\title{
An energy saving method of stable control of inverted pendulum system when affected by external interference using auxiliary pendulum
}

\author{
Chiem Nguyen ${ }^{1, *}$, Hai Phan $^{2}$, and Hung Nguyen ${ }^{2}$ \\ ${ }^{1}$ Department of Automation and Computing Techniques, Le Quy Don Technical University, Vietnam \\ ${ }^{2}$ Department of Software Engineering, Le Quy Don Technical University, Vietnam
}

\begin{abstract}
This research aims to develop a method to reduce energy consumption when controlling an inverted pendulum system that is affected by external interference. In this paper, the authors use the quasi time-optimal control law and add on an inverted pendulum an auxiliary pendulum to absorb the energy of the external interference effects, to reduce the cost of controlling the energy stable inverted pendulum while ensuring system quality. The quality of the method is demonstrated through simulation results. The effectiveness of this method is shown by comparison with the method of no damping.
\end{abstract}

\section{Introduction}

Inverted pendulum systems have many industrial and defense applications, for example: two-wheel self-balancing (seg-way) vehicles, missiles, self-propelled ammunition, smart robots, rigs and crane systems [10]. This is a multi-input and multiple-output mechanical system (MIMO) with high nonlinearity, instability [11]. On real objects, the cost of energy controlling the system is also an urgent issue to be researched. The works [1-4] use time optimal control laws for inverted pendulum systems, but with high energy costs, therefore are difficult fit in embedded systems. In the works [5-9], the authors have not introduced the energy saving problem, only interested the quality of the control system. In this paper, the authors presents a quasi time optimal control method and attaches on an inverted pendulum an auxiliary pendulum with elastic coefficient and dissipation coefficient to reduce system energy costs and control quality is not affected.

\section{The mathematical model of the inverted pendulum system and the inverted pendulum system with an auxiliary pendulum.}

Linear model of inverted pendulum system has been presented in works [2,3]. Assume that the friction force and moment of inertia of the bar are negligible. Nonlinear mathematical model of the pendulum system has the form:

\footnotetext{
*Corresponding author: nguyenxuanchiem83@gmail.com
} 


$$
\left\{\begin{array}{l}
\left(M+m_{1}\right) \ddot{x}+m_{1} d_{1} \ddot{\theta}_{1}=F \\
m_{1} d_{1} \ddot{x}+m_{1} d_{1}^{2} \ddot{\theta}_{1}-m_{1} g d_{1} \theta_{1}=0
\end{array}\right.
$$

In which: $x$ - cart position $(\mathrm{m}) ; \dot{x}$ - cart speed $(\mathrm{m} / \mathrm{s}) ; \ddot{x}$ - cart acceleration $\left(\mathrm{m} / \mathrm{s}^{2}\right) ; \theta_{1}$ deflection angle $(\mathrm{rad}) ; \ddot{\theta}_{1}$ - angular velocity $(\mathrm{rad} / \mathrm{s}) ; \ddot{\theta}_{1}$ - angular acceleration $\left(\mathrm{rad} / \mathrm{s}^{2}\right) ; F$ force $(N) ; k_{l}$ - friction coefficient $(N m / A) ; g$ - gravitational acceleration $\left(\mathrm{m} / \mathrm{s}^{2}\right) ; d_{l}$ pendulum length $(\mathrm{m}) ; M$ - cart weight $(\mathrm{kg}) ; \mathrm{m}_{\mathrm{l}}$ - weight of pendulum $(\mathrm{kg})$.

The inverted pendulum model is mounted on an inverted pendulum with an auxiliary pendulum illustrated in Figure 1.

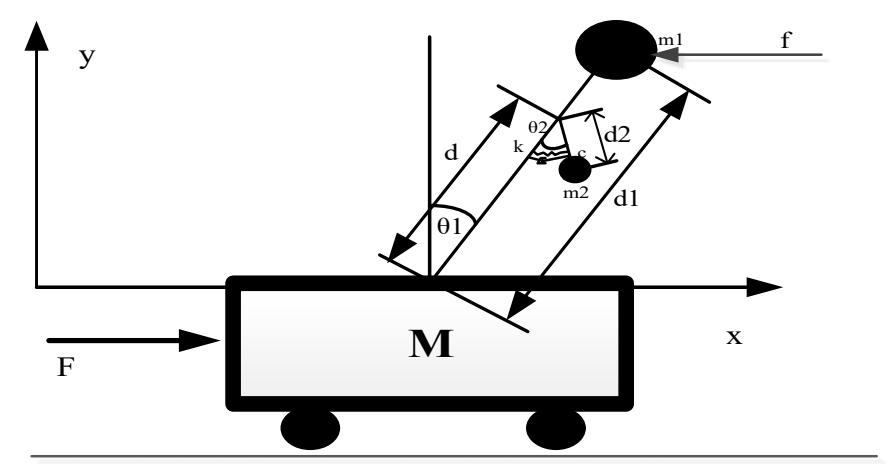

Fig. 1. Inverted pendulum system attaches additional auxiliary pendulum

In which the auxiliary pendulum has a elastic coefficient $k$ and dissipation coefficient $c$. Assume that the friction coefficient and moment of inertia of the bar are negligible. The model of linearization math at the point $(0,0,0)$ of the inverted pendulum system has an additional damping device of the form:

$$
\begin{cases}\left(M+m_{1}+m_{2}\right) \ddot{x}+\left(m_{1} d_{1}+m_{2} d-m_{2} d_{2}\right) \ddot{\theta}_{1}+m_{2} d_{2} \ddot{\theta}_{2} & =F \\ \left(m_{1} d_{1}+m_{2} d-m_{2} d_{2}\right) \ddot{x}+\left(m_{1} d_{1}^{2}+m_{2} d^{2}+m_{2} d_{2}^{2}-2 m_{2} d l_{2}\right) \ddot{\theta}_{1}+ & \\ +\left(m_{2} d d_{2}-m_{2} d_{2}^{2}\right) \ddot{\theta}_{2}-m_{1} g d_{1} \theta_{1}-m_{2} g d \theta_{1}-m_{2} g d_{2}\left(\theta_{2}-\theta_{1}\right) & =f \\ m_{2} d_{2} \ddot{x}+\left(m_{2} d d_{2}-m_{2} d_{2}^{2}\right) \ddot{\theta}_{1}+m_{2} d_{2}^{2} \ddot{\theta}_{2}+k \theta_{2}+m_{2} g d_{2}\left(\theta_{2}-\theta_{1}\right)+c \dot{\theta}_{2}=0\end{cases}
$$

In which: $x$ - cart position $(m) ; \dot{x}$ - cart speed $(\mathrm{m} / \mathrm{s}) ; \ddot{x}$ - cart acceleration $\left(\mathrm{m} / \mathrm{s}^{2}\right) ; \dot{\theta}_{1}, \dot{\theta}_{2}-$ deflection angle $(\mathrm{rad}) ; \dot{\theta}_{1}, \dot{\theta}_{2}$ - angular velocity $(\mathrm{rad} / \mathrm{s}) ; \ddot{\theta}_{1}, \ddot{\theta}_{2}$ - angular acceleration $\left(\mathrm{rad} / \mathrm{s}^{2}\right)$; $F$ - force $(N) ; f$ - interference affecting the pendulum $(N) ; k$ - elastic coefficient $(N / m) ; c$ dissipation coefficient $(N . \mathrm{s} / \mathrm{m}) ; g$ - gravitational acceleration $\left(\mathrm{m} / \mathrm{s}^{2}\right) ; d$ - length to point of auxiliary pendulum attachment $(m) ; d_{l}, d_{2}$ - pendulum lengths $(m) ; M$ - cart weight $(\mathrm{kg}) ; m_{l}$ - weight of main pendulum $(\mathrm{kg}), \mathrm{m}_{2}$ - weight of auxiliary pendulum $(\mathrm{kg})$.

\section{The synthesizing of quasi time-optimal control law for the pendulum systems}

The nearly optimal method by quick acts has been applied in some studies $[1,2,5]$. This method has many advantages such as fast duration effect, asymptotic stability, and high stability. In the paper [10], development of control rules on embedded systems with the one - free - step object is shown. Using a nonlinear model for the two - free - step inverted 
pendulum systems is slightly complicated. Therefore, the authors use the linear system (1) for embedded systems.

Changing state variables as follows:

$$
y_{1}=x_{1}+l x_{3} ; y_{2}=x_{2}+l x_{4} ; y_{3}=x_{3} ; y_{4}=x_{2}
$$

It is easy to verify that Jacobian matrix of transformation (4) is not degraded:

$$
\operatorname{det}\left[\frac{\partial y}{\partial X}\right]=\left|\begin{array}{llll}
1 & 0 & l & 0 \\
0 & 1 & 0 & l \\
0 & 0 & 1 & 0 \\
0 & 1 & 0 & 0
\end{array}\right|=-l \neq 0
$$

Where: $X=\left(\begin{array}{llll}x_{1} & x_{2} & x_{3} & x_{4}\end{array}\right)^{T}$.

Equations by $Y=\left(\begin{array}{llll}y_{1} & y_{2} & y_{3} & y_{4}\end{array}\right)^{T}$ :

$$
\left[\begin{array}{l}
\dot{y}_{1} \\
\dot{y}_{2} \\
\dot{y}_{3} \\
\dot{y}_{4}
\end{array}\right]=\left[\begin{array}{cccc}
0 & 1 & 0 & 0 \\
0 & 0 & g & 0 \\
0 & \frac{1}{l} & 0 & -\frac{1}{l} \\
0 & 0 & -\frac{m}{M} g & 0
\end{array}\right]\left[\begin{array}{l}
y_{1} \\
y_{2} \\
y_{3} \\
y_{4}
\end{array}\right]+\left[\begin{array}{c}
0 \\
0 \\
0 \\
\frac{1}{M}
\end{array}\right] F
$$

Equations (5) is in a controllable Jordan form [8]. Virtual system (5), which has asymptotic stablily and quasi - time optimum, is used to synthesize the control law F with $z_{1}$ $=y_{1} ; \mathrm{F}$ is found to introduce the system (5) into the virtual system (6) [8]. The obtained control law is a differentiable function of state variables, of which the formula is very long and therefore not given here.

$$
\left\{\begin{array}{l}
\dot{z}_{1}=-v \frac{z_{1}-x_{s p}}{\sqrt{\left(z_{1}-x_{s p}\right)^{2}+\varepsilon_{1}^{2}}}+z_{2} \\
\dot{z}_{2}=-\frac{z_{2}}{\varepsilon_{2}}+z_{3} \\
\dot{z}_{3}=-\frac{z_{3}}{\varepsilon_{3}}+z_{4} \\
\dot{z}_{4}=-\frac{z_{4}}{\varepsilon_{4}}
\end{array}\right.
$$

Where $h_{0}=\frac{z_{1}-x_{s p}}{\sqrt{\left(z_{1}-x_{s p}\right)^{2}+\varepsilon_{1}^{2}}}$ is quasi - time optimal function

$\left(h_{0}\left(z, x_{s p}\right) \approx \operatorname{sign}\left(z-x_{s p}\right)\right)[1]$. 


\section{Simulation results of an inverted pendulum system with auxiliary pendulum}

The selected parameters for the simulation model are as following : $M=100(\mathrm{~kg}), m=20$ $(\mathrm{kg}), l_{1}=20(\mathrm{~m}), g=9.81(\mathrm{~m} / \mathrm{s} 2), v=1.7, \varepsilon_{1}=0.35, \varepsilon_{2}=\varepsilon_{3}=\varepsilon_{4}=0.2, x_{s p}=0, x(0)=0(\mathrm{~m})$, $\dot{x}(0)=0(\mathrm{~m} / \mathrm{s}), \quad \theta_{1}(0)=0(\mathrm{rad}), \dot{\theta}_{1}(0)=0(\mathrm{rad} / \mathrm{s})$. The auxiliary inverted pendulum parameter is defined such that its length and mass are many times smaller than the length and parameter of the main inverted pendulum. In which: $\theta_{2}=0(\mathrm{rad}) ; \dot{\theta}_{2}=0(\mathrm{rad} / \mathrm{s}) ; k=$ $1000(\mathrm{~N} / \mathrm{m}) ; c=1000(\mathrm{~N} . \mathrm{s} / \mathrm{m}) ; d=20(\mathrm{~m}) ; d_{2}=1(\mathrm{~m}) ; m_{2}=2(\mathrm{~kg})$. In the simulation results, the authors selected the external interference as the signal with a cyclic form $f=M \sin (\omega t)(N)$. Simulation results and position and angle of inverted pendulum are shown in Figure 2a, 2b. On the graph in Figure 2a, it is clear that the position of the inverted pendulum system with the auxiliary pendulum (IPS + P) has a small amplitude of oscillation and overshoot much less than the conventional inverted pendulum system (IPS). The deflection angle of the pendulum with the auxiliary pendulum oscillates with negligible amplitude compared to the amplitude of the deflection angle of the conventional pendulum system (Figure $2 b)$.

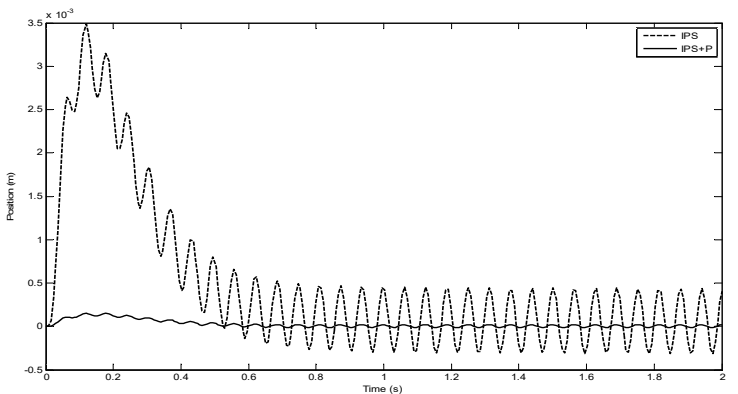

a)

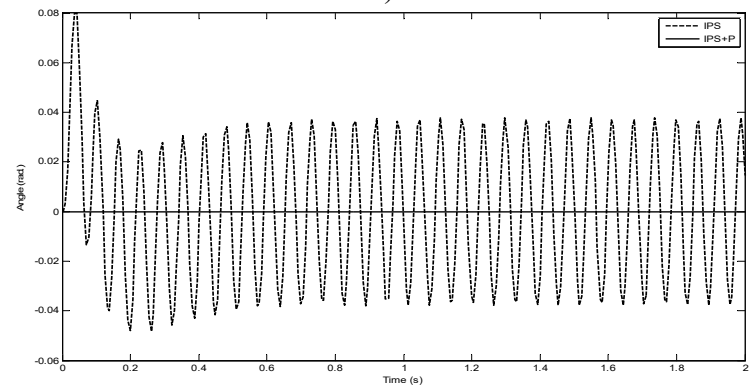

b)

Fig. 2. The position and angle response of the inverted pendulum system when affected by interfenrence.

The graph of Figure 3 shows the simulation results of the force acting on the inverted pendulum system when there is an external interference effect. It is easy to recognize that the cost of force acting on the inverted pendulum system is often much greater than the force cost acting on the inverted pendulum system with an attached auxiliary pendulum. The total cost of force acting on the system during the simulation time of $2 \mathrm{~s}$ is in sequence: $4769.6(\mathrm{~N})$ and $21.39(\mathrm{~N})$. This shows the efficiency of the proposed method of saving the cost of controlling the inverted pendulum system. 


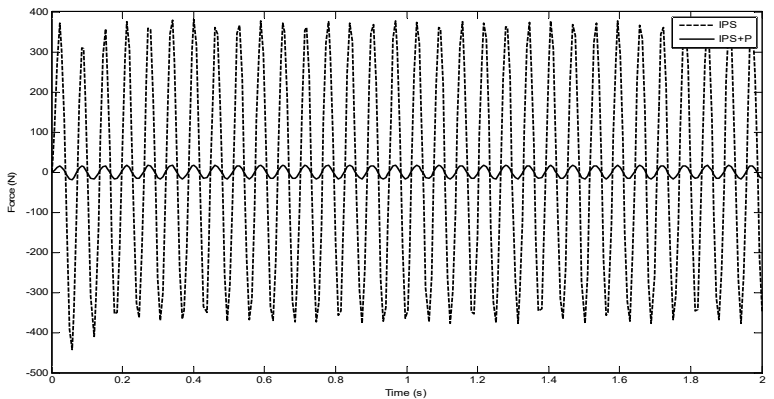

Fig. 3. The force response of the inverted pendulum system is affected by interfenrence

\section{Conclusion}

The paper presented a method to reduce energy costs for the inverted pendulum system using the quasi time-optimal optimal control law. It is the use of auxiliary pendulum mounted on the main inverted pendulum. Although there is a reduction in system dynamics, the pendulum will absorb the interference effect, increasing the stability of the system. The simulation results have shown that the quality of stable control of inverted pendulum system is well met. Specially reducing the system energy cost when stabilizing the inverted pendulum has the effect of external interfenrence.

\section{References}

1. R. Neydorf, Y. Sigida, V. Voloshin, Y. Chen, SAE Technical Paper 2013-01-2111, 2013, doi:10.4271/2013-01-2111.

2. H. N. Phan, C. X. Nguyen, MATEC Web Conf., 132, 02005 (2017)

3. H. Ohara, T. Murakami, A stability control by active angle control of front-wheel in a vehicle system, IEEE Trans. Ind. Electron, 55, 1277-1285 (2008).

4. C. N. Xuan, H. P. Nguyen, L. H. Duc, K. T. Dang, M. Le Kien, T. X. Pham, 2019 3rd International Conference on Recent Advances in Signal Processing, Telecommunications \& Computing (SigTelCom), 30 (2019)

5. L. B. Prasad, B. Tyagi, H. O. Gupta, International Journal of Automation and Computing, 11(6), 661 (2014)

6. S. Irfan, A. Mehmood, M. T. Razzaq, J. Iqbal, Engineering Science and Technology, an International Journal. In press, corrected roof, 21, 4, 753 (2018)

7. R. Bitirgen, M. Hancer, I. Bayezit, IFAC-PapersOnLine, 51, 4, 346 (2018)

8. L.B. Prasad, B. Tyagi, H.O. Gupta, International Journal of Automation and Computing, 11(6), 661 (2014)

9. S.C. Lin, C.C. Tsai, H.C. Huang, Proceedings of the 2009 IEEE International Conference on Systems, Man, and Cybernetics, 1965 (2009)

10. S. Irfan, A. Mehmood, M. T. Razzaq, J. Iqbal, Engineering Science and Technology, an International Journal, https://doi.org/10.1016/j.jestch.2018.06.010

11. M. Bettayeb, C. Boussalem, R. Mansouri, U. Al-Saggaf, ISA Trans., 53, 508 (2014) 